\title{
More than fishing in the dark: PCR of a dispersed sequence produces simple but ultrasensitive Wolbachia detection
}

\author{
Daniela I Schneider ${ }^{1}$, Lisa Klasson², Anders E Lind ${ }^{2}$ and Wolfgang J Miller ${ }^{1 *}$
}

\begin{abstract}
Background: Detecting intracellular bacterial symbionts can be challenging when they persist at very low densities. Wolbachia, a widespread bacterial endosymbiont of invertebrates, is particularly challenging. Although it persists at high titers in many species, in others its densities are far below the detection limit of classic end-point Polymerase Chain Reaction (PCR). These low-titer infections can be reliably detected by combining PCR with DNA hybridization, but less elaborate strategies based on end-point PCR alone have proven less sensitive or less general.

Results: We introduce a multicopy PCR target that allows fast and reliable detection of A-supergroup Wolbachia - even at low infection titers - with standard end-point PCR. The target is a multicopy motif (designated ARM: A-supergroup repeat motif) discovered in the genome of wMel (the Wolbachia in Drosophila melanogaster). ARM is found in at least seven other Wolbachia A-supergroup strains infecting various Drosophila, the wasp Muscidifurax and the tsetse fly Glossina. We demonstrate that end-point PCR targeting ARM can reliably detect both high- and low-titer Wolbachia infections in Drosophila, Glossina and interspecific hybrids.
\end{abstract}

Conclusions: Simple end-point PCR of ARM facilitates detection of low-titer Wolbachia A-supergroup infections. Detecting these infections previously required more elaborate procedures. Our ARM target seems to be a general feature of Wolbachia A-supergroup genomes, unlike other multicopy markers such as insertion sequences (IS).

Keywords: Wolbachia, Drosophila, Glossina, Hybrid, High- and low-titer endosymbiont infection, Limit of detection, A-supergroup repeat motif (ARM)

\section{Background}

Detecting endosymbionts such as the widespread alphaproteobacterium Wolbachia in its host cell environment requires reliable and ideally simple but still sensitive molecular marker systems. When such bacteria are present at high titers, classic end-point PCR is sufficient to unambiguously determine infection status of an unknown specimen. Particularly for Wolbachia, a quite comprehensive set of diagnostic PCR markers has been developed and applied successfully. The most commonly used among these makers is the multi locus sequence typing (MLST) system [1-3] and the four hypervariable regions (HVRs) of the Wolbachia outer surface protein gene wsp

\footnotetext{
* Correspondence: wolfgang.miller@meduniwien.ac.at

'Laboratory of Genome Dynamics, Department of Cell and Developmental Biology, Center for Anatomy and Cell Biology, Medical University of Vienna, Waehringerstrasse 10, Vienna 1090, Austria

Full list of author information is available at the end of the article
}

[4,5]. Both MLST, comprising a set of five singlecopy Wolbachia genes, and the wsp locus were demonstrated to be highly useful for Wolbachia infection determination and consequent diversity assessment. However, those marker systems are limited if the endosymbiont persists at very low titers within the host, either only during a certain ontogenetic stage [6] or throughout all life stages. In both cases proper detection of the endosymbiont is hindered and this points towards the need of an alternative strategy for efficient, robust and fast Wolbachia detection. One approach to address this issue is to use multicopy Wolbachia gene markers for PCR analyses. Particularly insertion sequences (IS; $[7,8]$ ) represent a good strategy to increase the detection threshold $[9,10]$. However, this approach relies on the conservation of such elements and their copy-numbers in diverse strains, which might not be the case over longer evolutionary distances due to the mobile nature of these elements. Another 
approach to cope with the detection problem introduced by low-titer infections is 'nested PCR'. This method might help to increase the detection threshold but is also highly prone to contamination [6]. A third strategy combines standard PCR with consequent hybridization $[6,11,12]$, which increases overall detection limit by four orders of magnitude [6]. On the other hand, this is an elaborate and time-consuming technique. Hence, we set out to find a more sensitive marker for detection of low-titer Wolbachia infections using standard PCR and identified ARM as such a simple but 'ultra-sensitive' marker for A-supergroup Wolbachia.

\section{Results and discussion}

Identification of a multicopy marker associated with tandem repeats in A-supergroup Wolbachia genomes (ARM)

To find a marker that serves a highly sensitive detection method of low-titer Wolbachia strains we identified multicopy regions in the A-supergroup $w$ Mel genome (Wolbachia of Drosophila melanogaster; GenBank NC_002978). An intergenic region of $440 \mathrm{bp}$ associated with the recently described hypervariable tandem repeat region (Figure 1; [13]) was the most promising candidate, hereafter called ARM ( $A$-supergroup repeat motif) as it was found in 24 almost identical copies dispersed throughout the $w \mathrm{Mel}$ genome (Additional file 1). However, for a marker to be useful as a general tool it also needs to be conserved and present in multiple copies in other strains and we therefore used the $w \mathrm{Mel}$ repeat sequence to search an additional 13 draft and complete Wolbachia genomes from four different Wolbachia supergroups for the same sequence. We were able to identify the presence of the repeat in seven A-supergroup Wolbachia genomes ( $w \mathrm{Ha}$, $w \mathrm{Ri}, w \mathrm{Wil}, w \mathrm{Ana}, w \mathrm{Uni}$, $w$ Suzi and $w \mathrm{Gmm}$; see Table 1 ), albeit in variable copy numbers. In the Drosophila associated Wolbachia strains, the copy numbers were around 20 per genome (Table 1), whereas the other two A-supergroup genomes ( $w \mathrm{Uni}$ and $w \mathrm{Gmm}$ ) contained about half the amount of copies. Low number of hits in $w$ Uni is most likely explained by the incomplete status of the genome resulting in an underestimation of the actual copy number. In the B- ( $w$ No, $w$ VitB, $w$ Pip), $\mathrm{C}-(w \mathrm{Oo}, w \mathrm{Ov})$, and $\mathrm{D}$-supergroup $(w \mathrm{Bm})$ genomes, ARM was not found. Even though some of the genomes in supergroups B, C, and D are incomplete, the total absence of the repeat in all genomes from these supergroups suggests that this motif might be Wolbachia A-supergroup specific. Additionally, VNTR-tandem repeats associated with ARM in A-supergroup infections are also absent

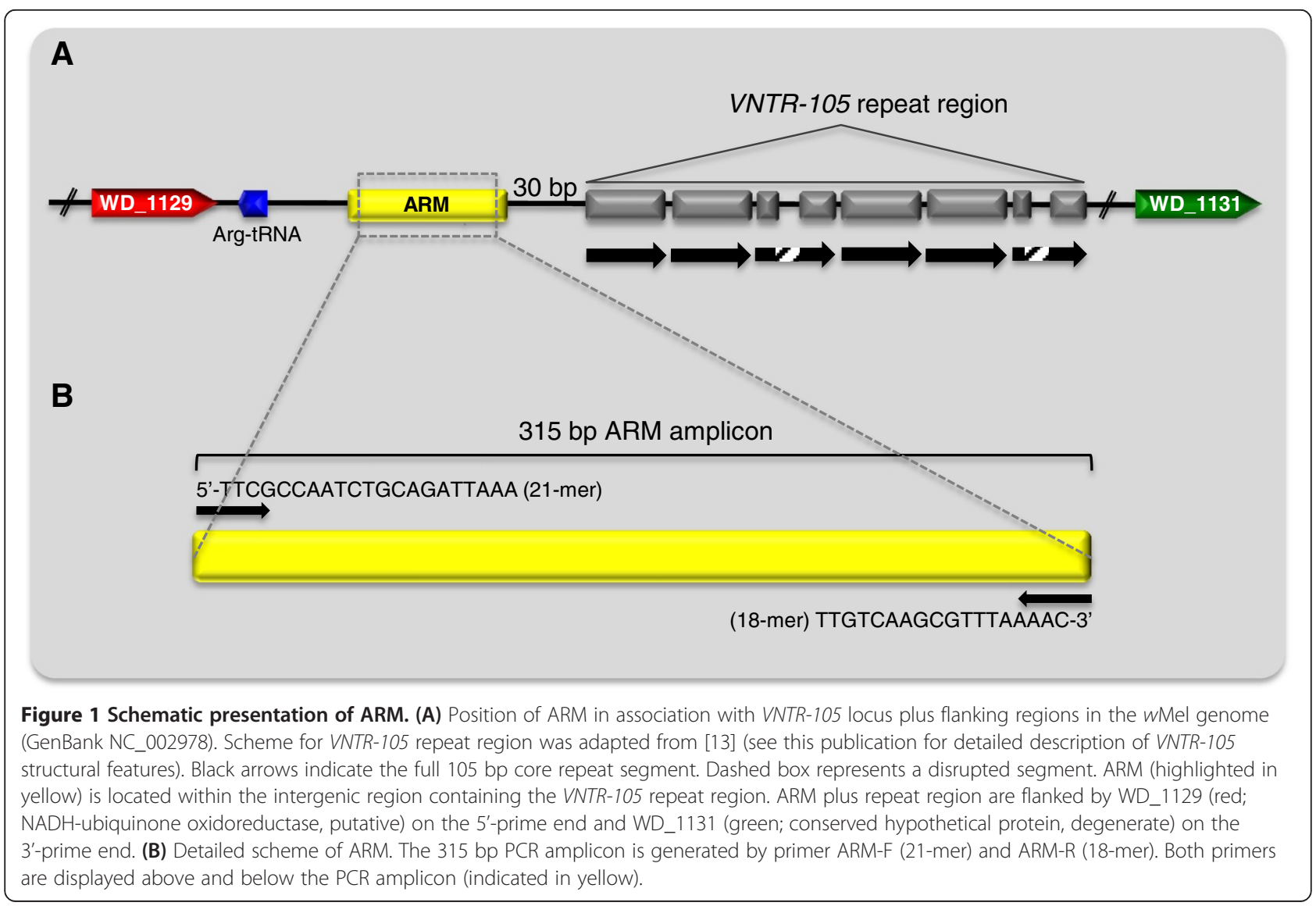


Table 1 Number of matches to ARM in complete and draft Wolbachia genomes

\begin{tabular}{|c|c|c|c|c|}
\hline Wolbachia & Supergroup & Host & Number of matches to $A R M$ & GenBank references \\
\hline wMel & A & Drosophila melanogaster & 24 & NC_002978; [8] \\
\hline$w \mathrm{Ha}$ & A & Drosophila simulans & 23 & CP003884; [23] \\
\hline$w \mathrm{Ri}$ & A & Drosophila simulans & 21 & NC_012416; [22] \\
\hline$w W i l$ & A & Drosophila willistoni & $17^{a}$ & ASM15358v1; TSC\#14030-0811.24 \\
\hline wAna & $\mathrm{A}$ & Drosophila ananassae & $20^{a}$ & ASM16747v1; [24] \\
\hline wUni & A & Muscidifax uniraptor & $7^{a}$ & wUni_1.0; [22] \\
\hline wSuzi & A & Drosophila suzukii & $23^{a}$ & CAOU02000000; [25] \\
\hline wGmm & A & Glossina morsitans morsitans & $20^{a}$ & [14] \\
\hline wNo & B & Drosophila simulans & $0^{\mathrm{b}}$ & CP003883; [23] \\
\hline$w$ VitB & B & Nasonia vitripennis & $0^{b}$ & WVB_1.0; [26] \\
\hline wPip & B & Culex quinquefasciatus & $0^{b}$ & NC_010981.1; [27] \\
\hline wOo & C & Onchocerca ochengi & $0^{\mathrm{b}}$ & NC_018267.1; [28] \\
\hline wOv & C & Onchocerca volvulus & $0^{\mathrm{b}}$ & ASM33837v1; [29] \\
\hline$w \mathrm{Bm}$ & $\mathrm{D}$ & Brugia malayi & $0^{\mathrm{b}}$ & NC_006833.1; [30] \\
\hline
\end{tabular}

Number of matches in column four refer to hits of the 315 bp ARM-PCR amplicon in the searched Wolbachia genomes. Hits were produced using the blastn algorithm (megablast) with match/mismatch scores 1,-2. Wolbachia strains are organized by supergroup (column two). Matches to ARM were only found within the A-supergroup. ${ }^{a}$ Minimum number of ARMs in the corresponding genome. Exact number cannot be given due to the lack of a complete genome. ${ }^{b}$ Refers to no similarity detected between ARM and searched genome (complete/draft).

from genomes of B- to D-supergroups, further indicating that this feature might indeed be A-supergroup specific.

\section{ARM facilitates detection of low-titer Wolbachia from A-supergoup}

ARM-targeting primer were tested via end-point PCR screen on DNA from high- and low-titer Wolbachia infections in Drosophila and Glossina (tsetse fly) species (Additional file 2). As shown in Figure 2, the classic Wolbachia singlecopy gene marker wsp (Wolbachia outer surface protein gene) is only applicable for samples with high-titer infections, since Wolbachia was only detected in high-titer $D$. paulistorum Orinocan semispecies (OR, Figure 2A) as well as in D. willistoni $\left(D w^{+}\right.$, Figure $\left.2 \mathrm{~B}\right)$, D. melanogaster $\left(\mathrm{Dm}^{+}\right.$, Figure $\left.2 \mathrm{~B}\right), \mathrm{D}$. simulans $\left(\mathrm{Ds}^{+}\right.$, Figure 2B) and Glossina morsitans morsitans (Gmm, Figure 2B). The wsp primer failed to detect Wolbachia in low-titer strains like D. paulistorum Amazonian (AM) and Centroamerican (CA) semispecies plus Glossina swynnertoni (Figure $2 \mathrm{~A}, \mathrm{~B}$ ), indicating that a singlecopy gene like $w s p$ is not suited for tracking low-titer infections. As multicopy gene markers like insertion sequences (IS) can be used to increase the detection limit, we ran PCR using primer for Insertion Sequence 5 (IS5; [8-10] on the same sample set. We observed increased sensitivity compared to wsp-PCR since Wolbachia was detected in low-titer CA2 (Figure 2A) and in the A/O hybrid samples. However, IS5 primer failed at amplifying the target sequence in all three Glossina samples (Gmm, Gsw and Gs/Gm hybrid; Figure 2B) despite the overall high Wolbachia titer in Gmm [12].
We have recently shown that Wolbachia titers increase in D. paulistorum [11] and Glossina [12] hybrid backgrounds, which should significantly facilitate detection and strain characterization. Such titer increase was sufficient to detect Wolbachia with the IS5 primer set in A/O hybrids, but the low-titer Wolbachia infection in the AM mother still remained undetected (Figure 2B). Failure of IS5-amplification in the Gs/Gm hybrid plus parents is explained by lacking homology between primer sequences and target, as no matches with the IS5 primer sequence were found in the $w \mathrm{Gmm}$ genome [14]. This finding implies that IS5 is not suitable as a general Wolbachia A-supergroup marker.

Figure 2A and B show that the ARM-marker system can be applied to address aforementioned problems arising with wsp and IS5 primer: sensitivity during PCR is increased significantly and all tested A-supergroup infections are unambiguously detected. Wolbachia was traced in all low-titer New world Drosophila species (AM1, AM2; CA1, CA2) plus the A/O hybrid. In contrast to IS5, the ARM primer set amplified Wolbachia from all three Glossina samples (Gmm, Gsw and Gs/Gm hybrid). As anticipated, all samples from high-titer Wolbachia infections (OR, $D w^{+}, D m^{+}, D s^{+}$) showed bright bands with ARM, whereas Wolbachia-uninfected specimens $\left(D w^{-}, D s^{-}\right)$did not (Figure 2A,B). This argues for a high specificity of the ARM primer and against mis-amplification of a random host target rather than the specific symbiont target site.

\section{Conclusions}

We suggest that the new multicopy Wolbachia Asupergroup marker can be used as an 'ultra-sensitive' 

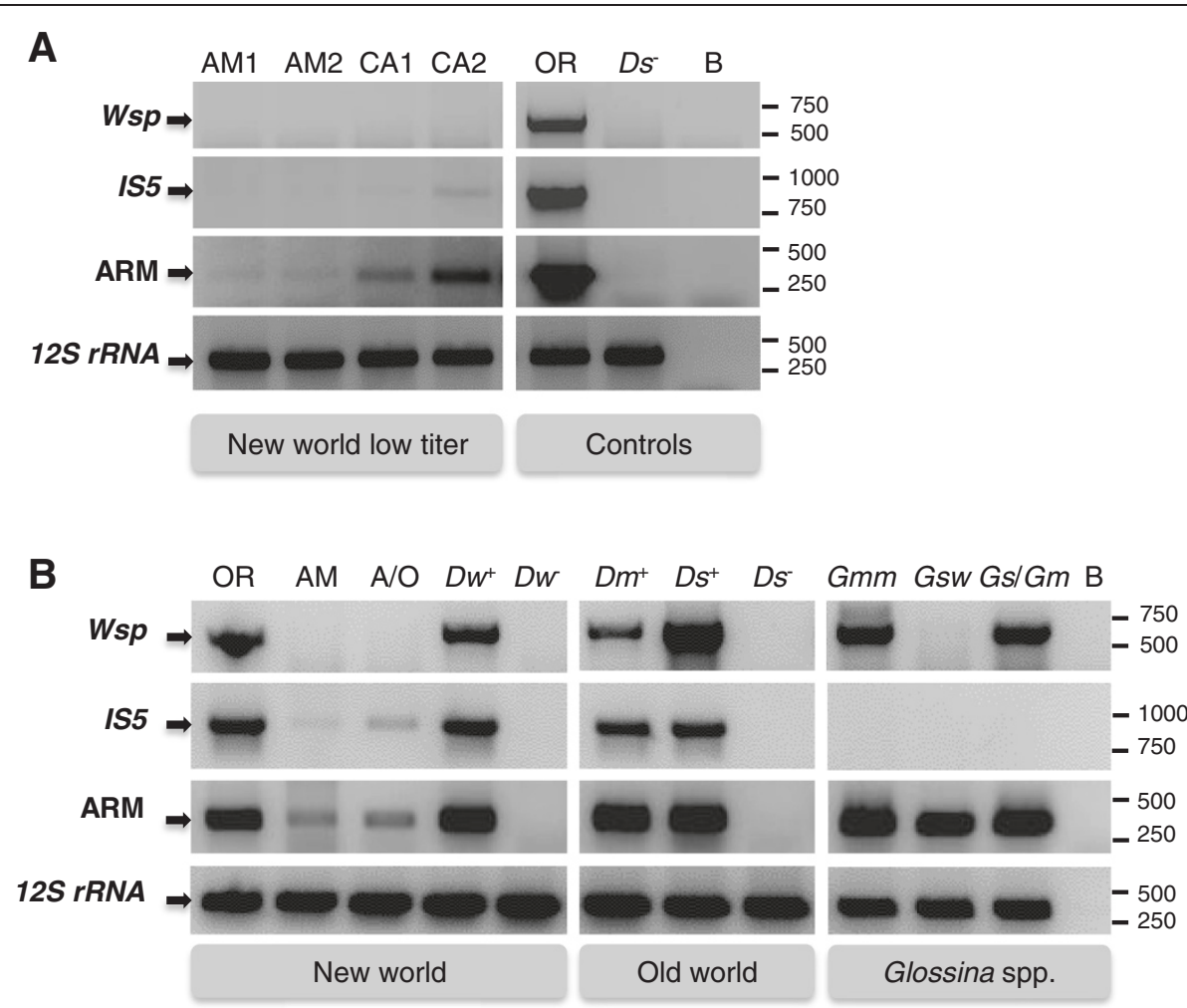

Figure 2 Comparison of Wolbachia marker sensitivity by PCR. (A) The three Wolbachia markers wsp, IS5 and ARM were tested on the following specimens: New world Drosophila species from the Drosophila willistoni group including D. paulistorum Amazonian (AM1, AM2), and Centroamerican (CA1, CA2) semispecies. Orinocan semispecies (OR) served as Wolbachia positive control; Ds as Wolbachia negative control. $\mathrm{B}=$ blank. Quality of DNA was assessed with universal primer set 12SCFR, 12SCRR targeting the mitochondrial 12S rRNA gene [20,21]. Expected amplicon sizes for Wolbachia positive control (OR) are 631 bp (wsp), 752 bp (IS5), 315 bp (ARM) and 399 bp (12S rRNA). (B) Same markers as above were tested on additional samples including hybrids: A/O hybrid plus parents AM and OR; Glossina Gs/Gm hybrid plus parental strains Gsw and Gmm (Additional file 2). Drosophila New world members include D. willistoni $D w^{+}$and Dw. Old world species are D. melanogaster Dm ${ }^{+} ; D$. simulans $D s^{+}$and $D s^{-}$. B = blank. Note: IS5 primer set does not produce amplicons in all three Glossina samples due to complete absence of this IS element in symbionts of tsetse flies (see discussion).

tool to trace low-titer infections by means of classic end-point PCR. First, ARM has the advantage of higher sensitivity compared to classic singlecopy Wolbachia markers like wsp and thus improves detection limit significantly. Particularly, ARM-PCR can be easily applied to screen larger numbers of untyped DNA specimens, even of low quality arising from long-term storage and/ or storage in inappropriate media, from laboratory stocks or samples directly from nature. This is of pivotal interest since classical detection tools might yield false negatives when examining species harboring Wolbachia at very low densities, and thereby lead to underestimating natural prevalence of A-supergroup infections. Given that $80 \%$ of the Dipteran infections are supergroup A [15], our new method will significantly facilitate and improve the sensitivity of such surveys. In addition our approach is an advantage over the classic IS5-marker, which fails in Wolbachia from the tsetse fly Glossina. Taken together, we show that a Wolbachia sequence motif found in multiple copies associated with the VNTR loci facilitates reliable Wolbachia screening of samples from low-titer infections and might thus serve as a great tool for the Wolbachia research community. Furthermore a similar approach might be applied to detect other symbionts such as Sodalis glossinidius (secondary symbiont of Glossina) and the primary symbiont Candidatus Sodalis pierantonius str. SOPE of the weevil Sitophilus orizae. Both symbiont genomes exhibit more than $20 \%$ of repetitive DNA rendering them appropriate candidates for repeatbased PCR analysis $[16,17]$. However, we anticipate that such a method reaches its limit when dealing with symbiont genomes, which have become highly streamlined in the course of tight host-symbiont coevolution.

\section{Methods}

Drosophila and Glossina strains plus hybrid samples

Drosophila specimens included members of New world and Old world clades (Additional file 2). Representatives of the new world clade were Drosophila paulistorum semispecies AM, CA and OR, together with Wolbachia- 
infected $\left(D w^{+}\right)$and -uninfected $\left(D w^{-}\right) D$. willistoni (see Additional file 2 for details). The Old world clade was represented by Wolbachia-infected D. melanogaster $\left(\mathrm{Dm}^{+}\right)$ and Wolbachia-infected $\left(D s^{+}\right)$and uninfected $\left(D s^{-}\right) D$. simulans (Additional file 2). Additionally, the tsetse fly species Glossina swynnertoni and G. morsitans morsitans (genus Glossina, superfamily Hippoboscoidea) and hybrids from D. paulistorum (A/O) and Glossina (Gs/Gm) were included (Additional file 2). Detailed descriptions of establishing hybrid samples can be found in $[11,12]$. Drosophila strains are permanently maintained in the Laboratory of Genome Dynamics in Vienna, Glossina colonies are kept at the Insect Pest Control Laboratory, Joint FAO/IAEA Division of Nuclear Techniques in Food and Agriculture, Vienna, Austria.

\section{Analysis of complete and draft Wolbachia genomes for candidate marker loci and primer design}

Candidate multicopy marker regions were identified by running nucmer and repeat-match from the MUMmer 3 package [18] on the wMel genome (Wolbachia, endosymbiont of Drosophila melanogaster; GenBank reference NC_002978). Searches were performed with the megablast algorithm using default settings against 14 Wolbachia genomes present in GenBank (see Table 1; www.ncbi.nlm. nih.gov) and other analyses were performed using Geneious 5.6.6 software (Biomatters, New Zealand).

\section{Diagnostic wsp-, IS5-, ARM- and 12S rRNA-PCR}

Primer pairs for diagnostic $w s p$-PCR were taken from [19] and the corresponding PCR set-up is described in [11]. Primers and PCR profile for IS5 can be found in [9]. We designed the following primer set targeting ARM: ARM-F 5'TTCGCCAATCTGCAGATTAAA-3' and ARM-R 5'GTTTTAAACGCTTGACAA-3'. Both primers are positioned in the flanking regions of the VNTR-105 locus in $w$ Mel $[9,13]$, and produce an amplicon of 315 bp constant size. Composition of the locus is shown in Figure 1. Diagnostic ARM-PCR was performed in $20 \mu \mathrm{l}$ reactions containing $1 \mathrm{x}$ reaction buffer, $3.0 \mathrm{mM} \mathrm{MgCl}_{2}, 0.4 \mu \mathrm{M}$ of forward and reverse primer, $35 \mu \mathrm{M}$ dNTPs, $0.4 \mathrm{U}$ of Taq Polymerase (Promega) and $2 \mu \mathrm{l}$ of DNA template. PCR was performed using a profile of $2 \mathrm{~min}$ initial denaturation at $94^{\circ} \mathrm{C}$ followed by 30 cycles consisting of $45 \mathrm{sec}$ denaturation at $94^{\circ} \mathrm{C}, 45 \mathrm{sec}$ annealing at $55^{\circ} \mathrm{C}$, and $1 \mathrm{~min}$ extension at $72^{\circ} \mathrm{C}$. Final extension was performed for $10 \mathrm{~min}$ at $72^{\circ} \mathrm{C}$. In order to assess DNA quality, we amplified part of the mitochondrial $12 S$ rRNA gene with primer set 12SCFR $5^{\prime}$-GAGAGTGACGGGCGATATGT$3^{\prime}$ and 12SCRR 5'-AAACCAGGATTAGATACCCTATTAT-3' [20]. PCR conditions are outlined in [21]. PCR amplicons were examined using gel-electrophoresis on a $1 \%$ agarose gel pre-stained with $0.05 \mathrm{mg}$ ethidium bromide.

\section{Ethics statement}

This study did not involve any subjects and materials that require approval by an ethics committee (human, vertebrate, regulated invertebrates). No genetically modified organisms were part of this study.

\section{Additional files}

\section{Additional file 1: Positions of ARM in the wMel and wRi genomes. Circular schemes of the wRi (Wolbachia symbiont of Drosophila simulans; NC_012416; [22]) and WMel genomes (Wolbachia, endosymbiont of D. melanogaster; NC_002978; [8]), showing that ARM (indicated by black bars) is equally dispersed throughout the genomes. \\ Additional file 2: Detailed information on Drosophila and Glossina specimens used in this study. First column refers to the abbreviated code used for each specimen in text, figures and figure legends. Last column lists reference and/or collector's name [31,11,32-34,12].}

\section{Abbreviations}

VNTR: Variable number of tandem repeats; wsp: Wolbachia outer surface protein gene; IS5: Insertion sequence element 5; ARM: A-supergroup repeat motif.

\section{Competing interests}

The authors declare that they have no competing interests.

\section{Authors' contributions}

DIS and WJM conceived the study. DIS, LK, AEL and WJM designed and performed the experiments. WJM provided material. DIS, LK, AEL and WJM analyzed the data. DIS, LK and WJM wrote the manuscript. All authors read and approved the final version of the manuscript.

\section{Acknowledgements}

We thank E. Kehrer and M. Leitner for careful maintenance of fly strains in the lab, A. G. Parker and A. M. M. Abd-Alla for providing Glossina material and S. Aksoy from Yale School of Public Health for sharing wGmm genome data. DIS and WJM were partly funded by research grant FWF P22634-B17 from the Austrian Science Fund (FWF).

\section{Author details}

'Laboratory of Genome Dynamics, Department of Cell and Developmental Biology, Center for Anatomy and Cell Biology, Medical University of Vienna, Waehringerstrasse 10, Vienna 1090, Austria. ${ }^{2}$ Department of Molecular Evolution, Cell and Molecular Biology, Science for Life Laboratory, Biomedical Centre, Uppsala, Sweden.

Received: 3 March 2014 Accepted: 30 April 2014 Published: 12 May 2014

\section{References}

1. Maiden MC, Bygraves JA, Feil E, Morelli G, Russell JE, Urwin R, Zhang Q, Zhou J, Zurth K, Caugant DA, Feavers IM, Achtman M, Spratt BG: Multilocus sequence typing: a portable approach to the identification of clones within populations of pathogenic microorganisms. Proc Natl Acad Sci U S A 1998, 95:3140-3145.

2. Paraskevopoulos C, Bordenstein SR, Wernegreen JJ, Werren JH, Bourtzis K: Toward a Wolbachia multilocus sequence typing system: discrimination of Wolbachia strains present in Drosophila species. Curr Microbiol 2006, 53:388-395.

3. Baldo L, Dunning Hotopp JC, Jolley KA, Bordenstein SR, Biber SA Choudhury RR, Hayashi C, Maiden MC, Tettelin H, Werren JH: Multilocus sequence typing system for the endosymbiont Wolbachia pipientis. Appl Environ Microbiol 2008, 72:7098-7110.

4. Zhou W, Rousset F, O'Neil S: Phylogeny and PCR-based classification of Wolbachia strains using wsp gene sequences. Proc Biol Sci 1998, 265:509-515. 
5. Braig HR, Zhou W, Dobson SL, O'Neill SL: Cloning and characterization of a gene encoding the major surface protein of the bacterial endosymbiont Wolbachia pipientis. J Bacteriol 1998, 180:2373-2378.

6. Arthofer W, Riegler M, Schneider D, Krammer M, Miller WJ, Stauffer C: Hidden Wolbachia diversity in field populations of the European cherry fruit fly, Rhagoletis cerasi (Diptera, Tephritidae). Mol Ecol 2009, 18:3816-3830.

7. Masui S, Kamoda S, Sasaki T, Ishikawa H: The first detection of the insertion sequence ISW1 in the intracellular reproductive parasite Wolbachia. Plasmid 1999, 42:13-19.

8. Wu M, Sun LV, Vamathevan J, Riegler M, Deboy R, Brownlie JC, McGraw EA, Martin W, Esser C, Ahmadinejad N, Wiegand C, Madupu R, Beanan MJ, Brinkac LM, Daugherty SC, Durkin AS, Kolonay JF, Nelson WC, Mohamoud Y, Lee P, Berry K, Young MB, Utterback T, Weidman J, Nierman WC, Paulsen IT, Nelson KE, Tettelin H, O'Neill SL, Eisen JA: Phylogenomics of the reproductive parasite Wolbachia pipientis wMel: a streamlined genome overrun by mobile genetic elements. PLOS Biol 2004, 2:E69.

9. Riegler M, Sidhu M, Miller WJ, O'Neill SL: Evidence for a global Wolbachia replacement in Drosophila melanogaster. Curr Biol 2005, 15:1428-1433.

10. Cordaux R: ISWpi1 from Wolbachia pipientis defines a novel group of insertion sequences within the IS5 family. Gene 2008, 409:20-27.

11. Miller WJ, Ehrman L, Schneider D: Infectious speciation revisited: impact of symbiont-depletion on female fitness and mating behavior of Drosophila paulistorum. PLoS Pathog 2010, 6:e1001214.

12. Schneider DI, Garschall KI, Parker AG, Abd-Alla AM, Miller WJ: Global Wolbachia prevalence, titer fluctuations and their potential of causing cytoplasmic incompatibilities in tsetse flies and hybrids of Glossina morsitans subgroup species. J Invertebr Pathol 2013, 112(Suppl):S104-S115.

13. Riegler M, Iturbe-Ormaetxe I, Woolfit M, Miller WJ, O'Neill SL: Tandem repeat markers as novel diagnostic tools for high resolution fingerprinting of Wolbachia. BMC Microbiol 2012, 12(Suppl 1):S12.

14. Brelsfoard C, Tsiamis G, Falchetto M, Gomulski L, Telleria E, Alam U, Ntountoumis E, Swain M, Malacrida A, Bourtzis K, Aksoy S: Wolbachia symbiont genome sequence and extensive chromosomal insertions described from the tsetse fly Glossina morsitans. 2014. in press: doi:10.1371/journal.pntd.0002728.

15. Stahlhut JK, Desjardins CA, Clark ME, Baldo L, Russell JA, Werren JH, Jaenike $\mathrm{J}$ : The mushroom habitat as an ecological arena for global exchange of Wolbachia. Mol Ecol 2010, 19:1940-1952.

16. Belda E, Moya A, Bentley S, Silva FJ: Mobile genetic element proliferation and gene inactivation impact over the genome structure and metabolic capabilities of Sodalis glossinidius, the secondary endosymbiont of tsetse flies. BMC Genom 2010, 11:449.

17. Oakeson KF, Gil R, Clayton AL, Dunn DM, von Niederhausern AC, Hamil C, Aoyagi A, Duval B, Baca A, Silva FJ, Vallier A, Jackson DG, Latorre A, Weiss RB, Heddi A, Moya A, Dale C: Genome degeneration and adaptation in a nascent stage of symbiosis. Genome Biol Evol 2014, 6:76-93.

18. Kurtz S, Phillippy A, Delcher AL, Smoot M, Shumway M, Antonescu C, Salzberg SL: Versatile and open software for comparing large genomes. Genome Biol 2004, 5:R12.

19. Jeyaprakash A, Hoy MA: Long PCR improves Wolbachia DNA amplification: wsp sequences found in $76 \%$ of sixty-three arthropod species. Insect Mol Biol 2000, 9:393-405.

20. Hanner R, Fugate M: Branchiopod phylogenetic reconstruction from $12 \mathrm{~S}$ rDNA sequence data. J Crustacean Biol 1997, 17:74-183.

21. Augustinos AA, Santos-Garcia D, Dionyssopoulou E, Moreira M, Papapanagiotou A, Scarvelakis M, Doudoumis V, Ramos S, Aguiar AF, Borges PA, Khadem M, Latorre A, Tsiamis G, Bourtzis K: Detection and characterization of Wolbachia infections in natural populations of aphids: is the hidden diversity fully unraveled? PLoS One 2011, 6:e28695.

22. Klasson L, Westberg J, Sapountzis P, Näslund K, Lutnaes Y, Darby AC, Veneti Z, Chen L, Braig HR, Garrett R, Bourtzis K, Andersson SG: The mosaic genome structure of the Wolbachia wRi strain infecting Drosophila simulans. Proc Natl Acad Sci U S A 2009, 106:5725-5730.

23. Elegaard KM, Klasson L, Näslund K, Bourtzis K, Andersson SG: Comparative genomics of Wolbachia and the bacterial species concept. PLOS Genet 2013, 9:e1003381.

24. Salzberg SL, Dunning Hotopp JC, Delcher AL, Pop M, Smith DR, Eisen MB, Nelson WC: Serendipitous discovery of Wolbachia genomes in multiple Drosophila species. Genome Biol 2005, 6:R23. Erratum in. Genome Biol 2005, 6:402.
25. Siozios S, Cestaro A, Kaur R, Pertot I, Rota-Stabelli O, Anfora G: Draft Genome Sequence of the Wolbachia Endosymbiont of Drosophila suzukii. Genome Announc 2013, 1:e00032-13. doi:10.1128/genomeA.00032-13.

26. Kent BN, Salichos L, Gibbons JG, Rokas A, Newton IL, Clark ME, Bordenstein SR: Complete bacteriophage transfer in a bacterial endosymbiont (Wolbachia) determined by targeted genome capture. Genome Biol Evol 2011, 3:209-218.

27. Klasson L, Walker T, Sebaihia M, Sanders MJ, Quail MA, Lord A, Sanders S, Earl J, O'Neill SL, Thomson N, Sinkins SP, Parkhill J: Genome evolution of Wolbachia strain wPip from the Culex pipiens group. Mol Biol Evol 2008, 25:1877-1887.

28. Darby AC, Armstrong SD, Bah GS, Kaur G, Hughes MA, Kay SM, Koldkjær P, Rainbow L, Radford AD, Blaxter ML, Tanya VN, Trees AJ, Cordaux R, Wastling JM, Makepeace BL: Analysis of gene expression from the Wolbachia genome of a filarial nematode supports both metabolic and defensive roles within the symbiosis. Genome Res 2012, 22:2467-2477.

29. Desjardins CA, Cerqueira GC, Goldberg JM, Chandler M, Mahillon J: Insertion sequences revisited. In Edited by Craig NL, Craigie R, Gellert M, Lambowitz AM. Edited by Mobile DNA II. Washington, DC: American Society of Microbiology; 2002:305-366.

30. Foster J, Ganatra M, Kamal I, Ware J, Makarova K, Ivanova N, Bhattacharyya A, Kapatral V, Kumar S, Posfai J, Vincze T, Ingram J, Moran L, Lapidus A Omelchenko M, Kyrpides N, Ghedin E, Wang S, Goltsman E, Joukov V, Ostrovskaya O, Tsukerman K, Mazur M, Comb D, Koonin E, Slatko B: The Wolbachia genome of Brugia malayi: endosymbiont evolution within a human pathogenic nematode. PLOS Biol 2005, 3:E121.

31. Ehrman L, Powell JR: The Drosophila willistoni species group. In Ashburner, Carson, Thompson; 1981-1986:193-225

32. Miller WJ, Riegler M: Evolutionary dynamics of wAu-like Wolbachia variants in Neotropical Drosophila species. Appl Environ Microbiol 2006, 72:826-835.

33. Kidwell MG, Novy JB: Hybrid dysgenesis in Drosophila melanogaster: sterility resulting from gonadal dysgenesis in the P-M system. Genetics 1979, 92:1127-1140.

34. Poinsot D, Montchamp-Moreau C, Merçot H: Wolbachia segregation rate in Drosophila simulans naturally bi-infected cytoplasmic lineages. Heredity (Edinb) 2000, 85(Pt 2):191-198.

doi:10.1186/1471-2180-14-121

Cite this article as: Schneider et al:: More than fishing in the dark: PCR of a dispersed sequence produces simple but ultrasensitive Wolbachia detection. BMC Microbiology 2014 14:121.

\section{Submit your next manuscript to BioMed Central and take full advantage of:}

- Convenient online submission

- Thorough peer review

- No space constraints or color figure charges

- Immediate publication on acceptance

- Inclusion in PubMed, CAS, Scopus and Google Scholar

- Research which is freely available for redistribution

Submit your manuscript at www.biomedcentral.com/submit
C Biomed Central 